\title{
Analysis of Channel Coding in Fading Channels for MIMO Systems
}

\author{
Umakanta Parida \\ College of Engineering and \\ Management, Kapurthala,
}

INDIA-144601

\author{
Sunil Kumar \\ College of Engineering and \\ Management, Kapurthala, \\ INDIA-144601
}

\author{
Omkar Singh \\ Lovely Professional University, \\ Punjab, \\ INDIA-144402
}

\begin{abstract}
Wireless communication systems are being designed to integrate features that include high data rates as well as high quality of service in the existing communication framework. For this an orthogonal space-time block code (OSTBC) technique improves error performance of synchronous data links without sacrificing data rate or requiring more bandwidth. Trellis coded modulation (TCM) enables efficient transmission scheme and to achieve high coding gain by integrating coding and modulation. In this work an OSTBC concatenated with TCM is implemented for information transmission over different antenna configurations, from Single-Input Single-Output (SISO) to Multi-Input MultiOutput (MIMO) channels. The channel used is a quasi-static Rayleigh fading channel with additive white Gaussian noise (AWGN). The proposed model takes the advantages of the concatenation scheme: the spatial diversity gain offered by OSTBC and the coding gain offered by TCM. Using MATLAB Simulink the system performance is compared with using only TCM or OSTBC and TCM concatenated with OSTBC. Significant performance improvement of the system is achieved using this scheme. The proposed scheme achieved full transmits diversity and coding gain of more than $2 \mathrm{~dB}$ without using interleavers/deinterleavers. It is observed that performance of MIMO system is better than SISO or MISO (Multi-Input SingleOutput) system.
\end{abstract}

\section{Keywords}

OSTBC, TCM, MIMO, SISO, AWGN, Interleaver, Concatenation

\section{INTRODUCTION}

Multi-input Multi-output (MIMO) systems with multiple antenna elements at both link ends are an efficient solution for wireless communications systems as they provide high data rates by exploiting the spatial domain under the constraints of limited bandwidth and transmit power. The main advantages of the MIMO system are array gain, interference reduction and diversity gain. In most scattering environments, antenna diversity is a practical, effective and, hence, a widely applied technique for reducing the effect of multipath fading [1]. Some other interesting approaches for transmit diversity are: spacetime trellis coding (STTC) [1], [2] and space-time block coding [3], [4]. Alamouti proposed a simple transmit diversity scheme which improves the signal quality at the receiver on one side of the link by simple processing across two transmit antennas on the opposite side [3]. This approach is later generalized to an arbitrary number of transmit antennas as space-time block coding [4].By efficiently integrating space and time domain, space-time trellis coding achieves full diversity of order $n_{r} \times n_{t}$ and substantial coding gain. [5]. in this paper, authors consider the utilization of multiple transmitter and receiver antennas for space-time diversity. The optimal SNR scheme, which also provides the best diversity, is outlined. This scheme however involves a reduction in the data rate. G. Ungerboeck described a coding technique which improves error performance of synchronous data links without sacrificing data rate or requiring more bandwidth. This is achieved by channel coding with expanded sets of multilevel/phase signals in a manner which increases free Euclidean distance [8]. In [10], a system concatenating STBC with TCM over fading channels is proposed and analyzed. The objectives of this work are to design an efficient concatenated scheme of OSTBC and TCM for MIMO system to achieve significant coding gain and diversity without using Interleaver/deinterleaver, hence to reduce the complexity and processing of the system.

\section{METHODS}

\subsection{Overview of MIMO}

Wireless communication is highly challenging due to the complex, time varying propagation medium. If we consider a wireless link with one transmitter and one receiver, the transmitted signal that is launched into wireless environment arrives at the receiver along a number of diverse paths, referred to as multi-paths. These paths occur from scattering and rejection of radiated energy from objects (buildings, hills, trees...) and each path has a different and time-varying delay, angle of arrival, and signal amplitude. As a consequence, the received signal can vary as a function of frequency, time and space. These variations are referred to as fading and cause deterioration of the system quality. Furthermore, wireless channels suffer of cochannel interference (CCI) from other cells that share the same frequency channel, leading to distortion of the desired signal and also low system performance. Therefore, wireless systems must be designed to mitigate fading and interference to guarantee a reliable communication. A successful method to improve reliable communication over a wireless link is to use multiple antennas.

\subsection{MIMO system (channel) model}

In this part a SISO ( $1 \times 1$, antenna configuration) system with only TCM, and the MIMO system with $2 \times 1,2 \times 2,3 \times 2$ transmit-receive antenna is modelled using the proposed concatenated scheme of OSTBC and TCM. This model takes the advantages of the concatenation scheme: the spatial diversity gain offered by OSTBC and the coding gain offered by TCM. For comparison, reference models containing only TCM or OSTBC are provided. The model is compared with different antenna configurations and different states of the TCM. The model is designed in the Simulink of MATLAB as shown in figure 1 . 


\subsubsection{Bernoulli Binary Generator}

This block generates random binary numbers using a Bernoulli distribution. If the Bernoulli distribution parameter is taken as $p$,

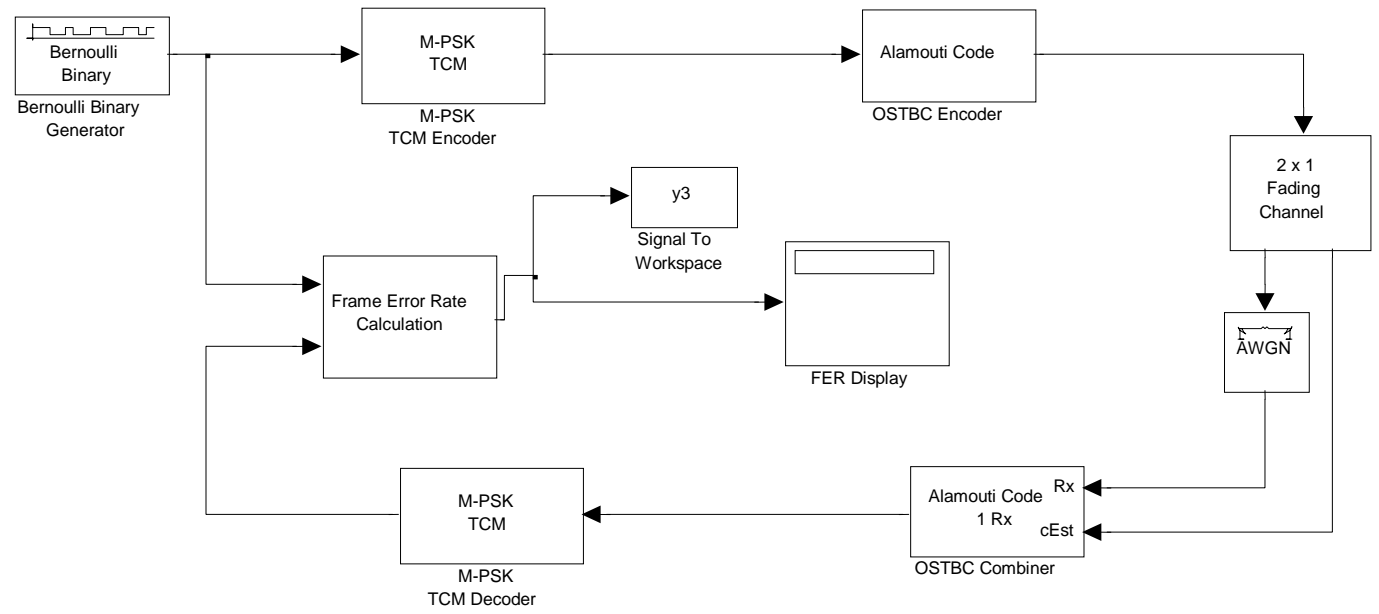

Fig 1: MIMO system model with both OSTBC and TCM

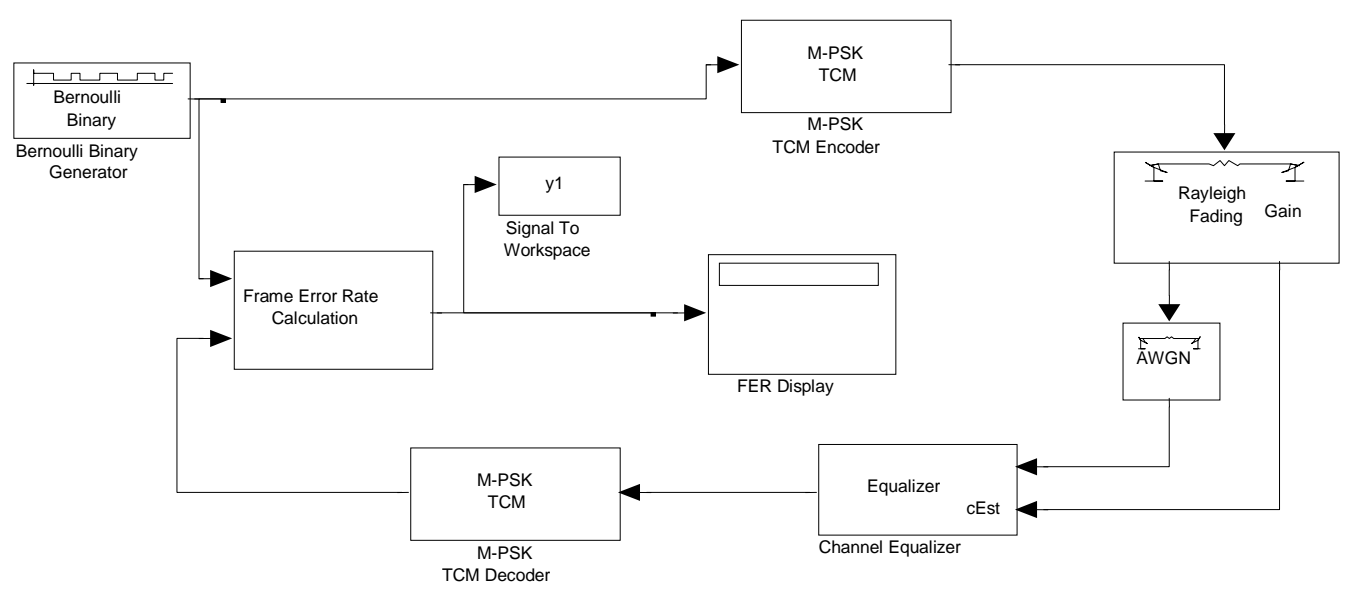

Fig 2: SISO system model with TCM only

it produces a, ' 0 ' with probability $\mathrm{p}$ and a' 1 ' with probability 1 $p$. The mean value and variance of the Bernoulli distribution is $1-p$ and $p(1-p)$. The Probability of a zero parameter specifies $p$, and can be any real number between zero and one. The output signal can be a frame-based matrix, a sample-based row or column vector, or a sample-based one-dimensional array. These attributes are controlled by the Frame-based outputs, Samples per frame, and Interpret vector parameters as 1-D parameters. In the model Bernoulli Binary Generator block produces the information source for this simulation. The block generates a frame of 100 random bits. The Samples per frame parameter determines the length of the output frame. In this case the length of the output frame is 100 and the output data type is "Boolean."

\subsubsection{Trellis-Coded Modulation Encoder}

In the M-PSK TCM Encoder block, TCM uses convolutional encoder to encode the binary input signal and maps the result to a PSK signal constellation. The M-ary number parameter is the number of points in the signal constellation, and the number of possible output symbols from the convolutional encoder. Here for a rate $\mathrm{k} / \mathrm{n}$ convolutional code $\mathrm{n}$ is, $\log 2(\mathrm{M}$-ary number). As the $\mathrm{M}$-ary number is set to 8 , it gives $\mathrm{n}=3$. If the convolutional encoder with the trellis structure gives a rate $\mathrm{k} / \mathrm{n}$ code, then the M-PSK TCM Encoder block's input must be a frame-based binary column vector whose length is $\mathrm{L}_{\mathrm{k}}$ for some positive integer L. The output from the M-PSK TCM Encoder block is a frame-based complex column vector of length $\mathrm{L}$. In this work the convolutional encoder, uses the Ungerboeck's trellis structure [8] with multilevel (amplitude and phase) modulation and the set partitioning concept. Using MATLAB's trellis structure function, different trellis structures are designed for an 8-state 8-PSK trellis by changing the "next state" and "output state" parameters. The structure with output states as given in (1) below, gives improved result. So this structure is an optimum for 
Ungerboeck's trellis structure. For the structure, number of input symbols, output symbols and states are as follow:

Number of input symbols $=4$

Number of output symbols $=8$

Number of states $=8$

Next states $=(8 \times 4$ double $)$

Outputs $=(8 \times 4$ double $)$

$\begin{array}{llll}0 & 4 & 2 & 6 \\ 4 & 0 & 6 & 2 \\ 2 & 6 & 0 & 4 \\ 6 & 2 & 4 & 0 \\ 1 & 5 & 3 & 7 \\ 5 & 1 & 7 & 3 \\ 3 & 7 & 1 & 5 \\ 7 & 3 & 5 & 1\end{array}$

The trellis-coded modulation technique partitions the constellation into subsets called cosets, to maximize the minimum distance between pairs of points in each coset. constellation diagram is shown in Figure. 3 In this M-PSK TCM block, the operation mode is set as Truncated (reset every frame) mode. In this mode, the block treats each frame independently. I.e., the encoder states are reset to all-zeros state at the start of each frame. The output type of the block can be specified as a single or double. Here it is set as double.

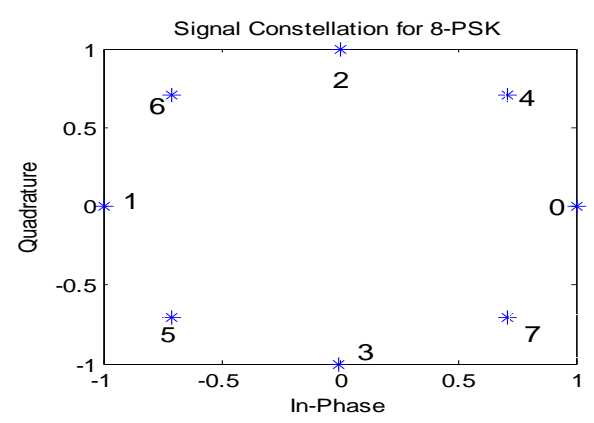

Fig. 3 Signal constellation for 8-PSK

Again a 4-state 8-PSK trellis structure is constructed for the comparison with 8-state 8-PSK trellis structure. Parameters for the 4-state trellis structure are taken as follows.

Number of input symbols $=4$

Number of output symbols $=8$

Number of states $=4$

Next states $=(4 \times 4$ double $)$

$$
\begin{array}{llll}
0 & 2 & 4 & 6 \\
1 & 3 & 5 & 7 \\
2 & 0 & 6 & 4 \\
3 & 1 & 7 & 5
\end{array}
$$$$
\text { Outputs }=(4 \times 4 \text { double })
$$

\subsubsection{M-PSK TCM Decoder}

The M-PSK TCM Decoder block uses the Viterbi algorithm to decode a trellis-coded modulation (TCM) signal that was formerly modulated using a PSK signal Constellation. The Mary number is the number of points in the signal constellation, and the number of probable output symbols from the convolutional encoder. The Trellis structure and $\mathrm{M}$-ary number parameters in this block should equal to those in the M-PSK TCM Encoder block, to guarantee accurate decoding. So it is set as 8. The input signal to this block is the output of the OSTBC combiner which is a frame-based column vector containing complex numbers. Decoder block's output is a frame-based binary column vector whose length is $\mathrm{k}$ times the vector length of the input signal. As the input signal length is 50, it gives output as $2 \times 50$ which is equal to 100 . The Operation mode factor is set to Truncated to treat each frame separately. The Trace back depth parameter, D is set to 25 . The trace back path starts at the state with the lowest metric. D must be less than or equal to the vector length of the input and $5 \sim 6$ times greater than the constraint length of the TCM. As the length of the input is 50 , and constraint length is $3, \mathrm{D}$ is taken as 25 to make sure an almost lossless performance.

\subsubsection{OSTBC Encoder}

This block encodes an input symbol sequence by means of orthogonal space-time block code (OSTBC). The block maps the input symbols block-wise and concatenates the output codeword matrices in the time domain. This block encodes the information symbols from the TCM Encoder by using the Alamouti code [3] for 2 transmit antennas.

\subsubsection{OSTBC Combiner}

The OSTBC combiner block takes the input signals from the receiving antennas. It combines the input signals and channel state information (CSI) to give the information about encoded symbols. The combining operation in the combiner is done separately for each symbol. This operation depends upon the number of transmit antennas and the coding rate.

\subsubsection{MIMO channel model}

In MIMO channel model we have designed different antenna configurations to increase the diversity gain of the system. One SISO (1 1, antenna configuration) is also given for comparison. In this model Multipath Rayleigh Fading Channel blocks are used. Rayleigh fading is a statistical model for the effect of propagation environment on a radio signal, such as used for wireless communication. It is most applicable where there is no dominant propagation along a line of sight between the transmitter and receiver. The main blocks used in the channel model are selector, Multipath Rayleigh fading channel, sum and matrix concatenate. The selector block gives the required order of the output to the Rayleigh fading channel. Then the output signal matrices from the channel are summed to produce a single stream and the path gain matrices are concatenated to give the channel estimates at the combiner. During simulation Additive White Gaussian noise (AWGN) is added at the receiver side.

\subsubsection{Calculation of Frame Error Rate (FER)}

Frame Error Rate is the ratio of data received with error to total data received per frame. In this block FER is calculated by comparing the original source bit taken from Bernoulli Binary Generator block with decoded bits from TCM decoder block. During simulation the simulation is checked by setting the parameters i.e. number of error frames or maximum number of frames which is earlier. Here maximum number of frame is taken as $5 \times 106$ and number of error frames as 103 . This block is then connected to "Signal to Workspace" block which saves the data in MATLAB workspace.

\section{RESULTS}

This section describes the MATLAB simulation results of the MIMO system. MATLAB is a high-level technical computing language and interactive environment for algorithm 
development, data visualization, data analysis, and numeric computation. The system is modeled using Simulink and all the graphs has been plotted through Monte Carlo Simulation in Bit Error Rate Analysis Tool (MATLAB), with respect to signal to noise ratio (SNR in $\mathrm{dB}$ ) in $\mathrm{X}$-axis and frame error rate (FER) in Y-axis. Figure 4 shows the Comparison of 8- state TCM with rearranged parameters. Here we have designed different 8-state trellis structures using Ungerboeck's structure by re-arranging the next states and output states. All the designed trellis structures are simulated in the MIMO system model. From the figure it is clear that the trellis structure in simulation-0 is best as compared to other structures. So we have used this trellis structure in our proposed concatenated scheme for further simulations. Figure 5 is a comparison between 8 -state and 4-state trellis. The 8 -state trellis gives a coding gain of $2 \mathrm{~dB}$ as compared to 4 -state trellis. So 8 -state trellis is more efficient, that is why used in our concatenated scheme. Figure 6 and Figure 7 shows the coding gain and diversity gain achieved through the proposed concatenated scheme. From the plot it is observed that the concatenation scheme of OSTBC and TCM gives improved and better result than only OSTBC or TCM. In figure 6, both the concatenated scheme and the uncoded scheme i.e. only OSTBC used a channel model of $2 \times 1$ antenna configuration. An $1 \times 1$ antenna configuration is used for TCM only. The concatenated scheme provides a diversity gain of 4 over the TCM scheme and more than $2 \mathrm{~dB}$ coding gain over OSTBC. In the model with only OSTBC, The TCM block is replaced by a QPSK modulation so that both the models have same symbol rate. Figure 8 shows the comparison of MIMO systems with different antenna configurations. The diversity gain of $3 \times 2$ antenna configuration with respect to $2 \times 2,2 \times 1,1 \times 1$ is 2,6 and 8 respectively.

\section{DISCUSSIONS}

As shown in the results, the proposed concatenated scheme of OCTBC and TCM gives significant improvement in terms of coding gain and diversity. Authors in reference [10] achieved more than $2 \mathrm{~dB}$ coding gain using perfect interleaver. But in our design the trellis structure is optimized to achieve the same gain without using the interleaver/deinterleaver. Hence reduces the complexity of the system model.

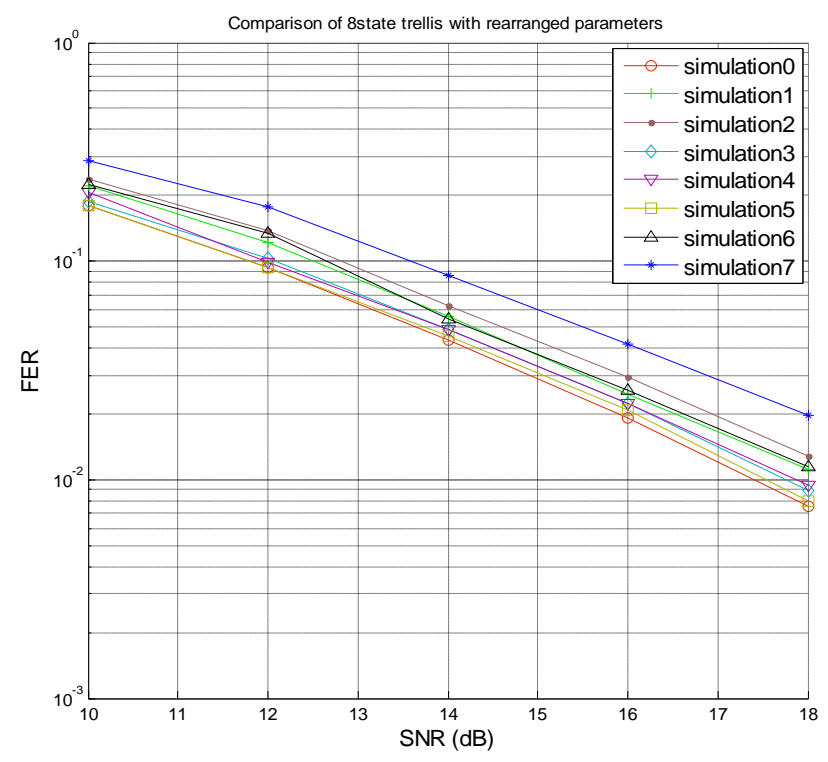

Fig 4: Comparison of 8state TCM with re-arranged parameters

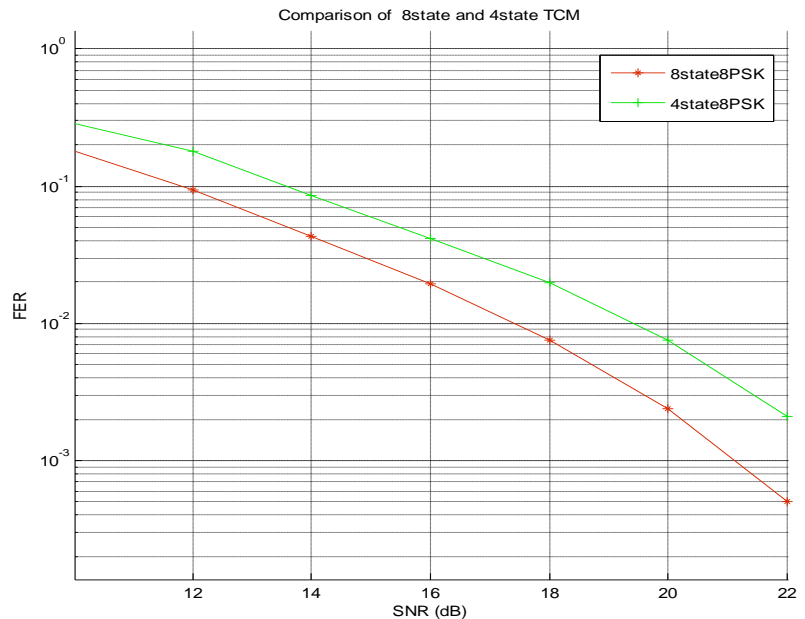

Fig 5: Comparison between 8state and 4state TCM

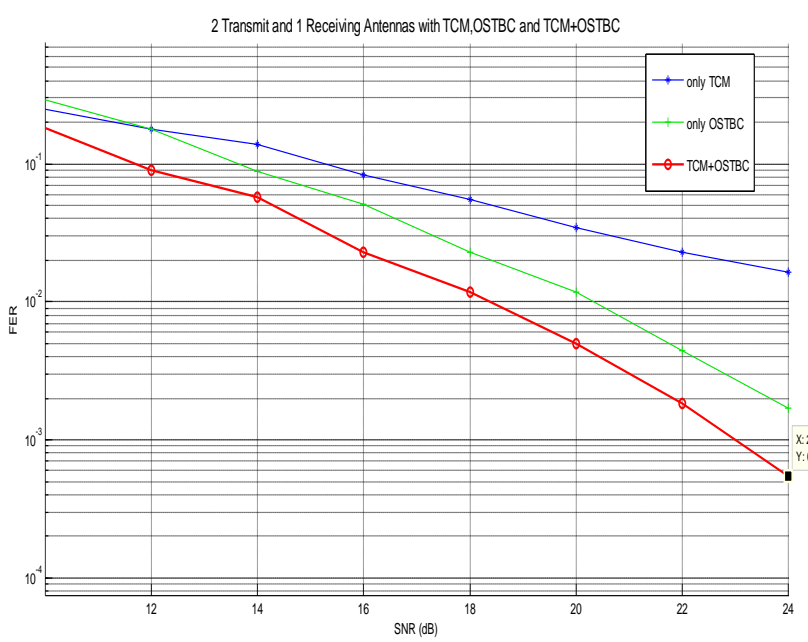

Fig 6: Comparison of the Concatenated scheme with only TCM or OSTBC

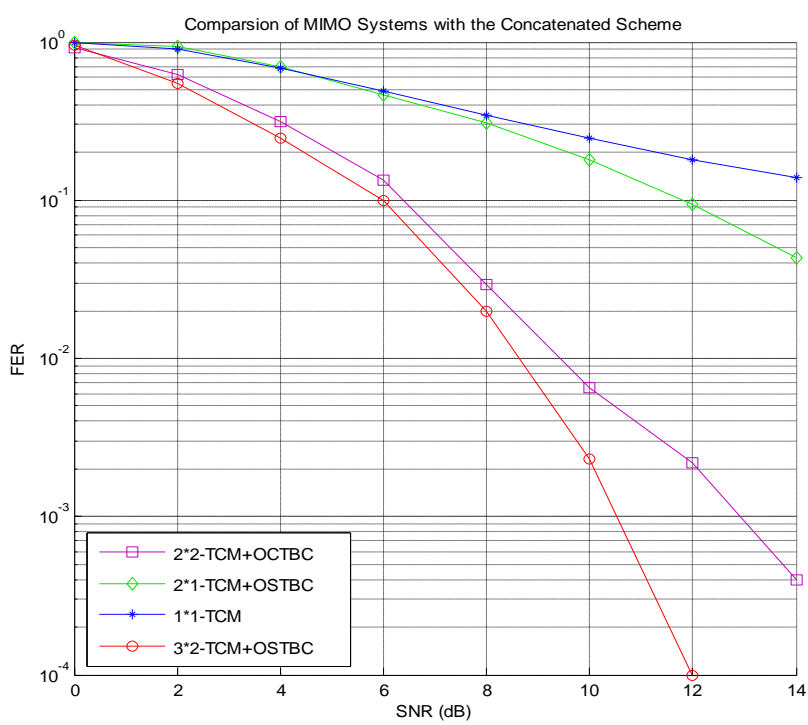

Fig 7: Comparison of MIMO systems with the concatenated scheme 


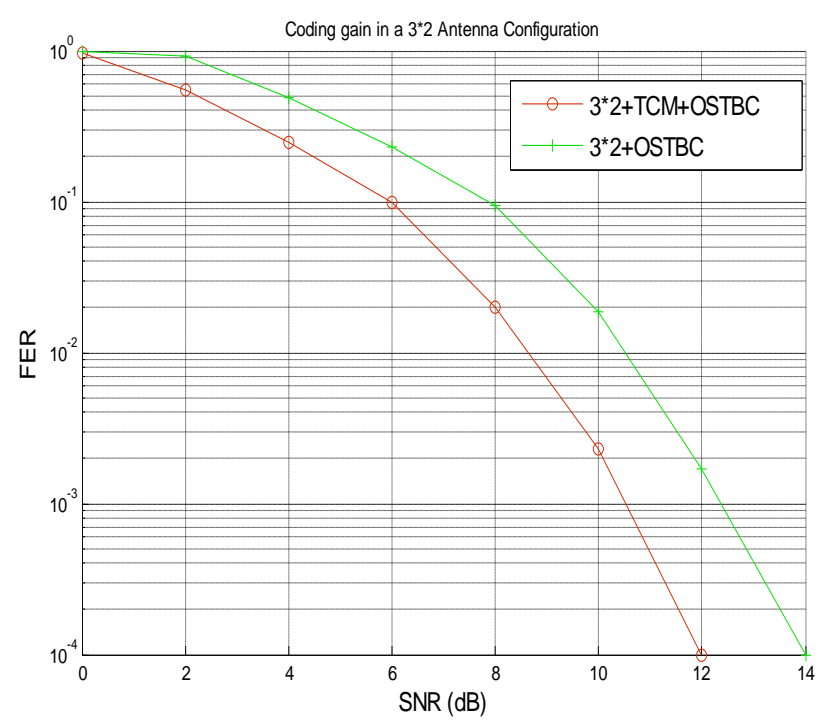

Fig 8: The Concatenated scheme in an $3 \times 2$ antenna configuration

\section{CONCLUSIONS}

In this work, a concatenated scheme of OSTBCs and TCM has been proposed which can be a good scheme for future wireless communication systems. The main disadvantage of OSTBCs were, for more than 2 transmit antennas and complex valued signals, OSTBCs only exist for code rates smaller than one symbol. A complex orthogonal design of OSTBCs which provides full diversity and full transmission rate is not possible for more than two transmit antennas. To get the full transmission rate a simple Alamouti code was used in the OSTBC with two transmit antennas. For more than two transmit antennas and to increase the transmission rate, design of new OSTBC techniques have a great scope for future work. The trellis structure used for the concatenated scheme is an 8state with 8PSK constellation mapping. For improvement in coding gain, higher state TCMs can be taken into consideration but as we will go for higher states, improvement in coding gain is not so significant. So optimal trellis design for higher state TCM is one of the scope for future research

\section{REFERENCES}

[1] Y. Gong and K. B. Letaief, "Performance Evaluation and Analysis of Space-Time Coding in Unequalized Multipath Fading Links," IEEE Trans. Commun., Vol. 48, No. 11, pp. 1778-1782, Nov. 2000.

[2] V. Tarokh, N. Seshadri, A. R. Calderbank, "Space-Time Codes for High Data Rate Wireless Communication: Performance Criterion and Code Construction", IEEE Trans. Inform. Theory, Vol. 44, No. 2, pp. 744-765, March 1998.

[3] S. M. Alamouti, "A simple transmit diversity technique for wireless communications," IEEE Journal on Selected Areas in Communications, Vol. 16, No. 8, pp. 1451-1458, Oct. 1998.

[4] V. Tarokh, H. Jafarkhami, and A. R. Calderbank, "Space-time block codes from orthogonal designs," IEEE Transactions on Information Theory, Vol. 45, No. 5, pp. 1456-1467, Jul. 1999.
[5] G. Ganesan, P.Stoica, "Space-time diversity using orthogonal and amicable orthogonal design", Wireless Personal Communications, Vol.18, pp.165-178, 2001.

[6] C. E. Shannon. A mathematical theory of communication. Bell System Technical Journal, 27: 379423 \& 623-656, July \& Oct. 1948.

[7] Stephen G. Wilson, "Digital Modulation and Coding," Prentice Hall, Inc., 1996.

[8] G. Ungerboeck, "Channel coding with multilevel/phase signals," IEEE Transactions on Information Theory, vol. IT-28, No. 1, pp. 55-67, Jan. 1982.

[9] S. M. Alamouti, V. Tarokh, and P. Poon, "Trellis-coded modulation and transmit diversity: Design criteria and performance evaluation," in Proceedings of IEEE International Conference on Universal Personal Communications (ICUPC'98), Florence, Italy, Vol. 1, pp. 703-707, Oct. 5-9, 1998.

[10] Y. Gong and K. B. Letaief, "Concatenated space-time block coding with trellis coded modulation in fading channels," IEEE Transactions on Wireless Communications, Vol. 1, No. 4, pp. 580-590, Oct. 2002.

[11] V. Tarokh, N. Seshadri and A.R. Calderbank, "SpaceTime Codes For Wireless Communication: Code Construction" AT\&T Labs-Research 600 Mountain Avenue Murray Hill, New Jersey 07974, IEEE 1997.

[12] G. Ungerboeck, "Trellis Coded Modulation with Redundant Signal Sets, Part I: Introduction," IEEE Communication Magazine, Vol. 25, No.2, pp. 5-11, February 1987.

[13] G. Ungerboeck, "Trellis Coded Modulation with Redundant Signal Sets, Part II: State of the Art", IEEE Communication Magazine, Vol. 25, No.2 pp. 12-21, February 1987.

[14] Olaf J. Joeressen and H. Meyr, "A $40 \mathrm{Mb} / \mathrm{s}$ Soft-output Viterbi Decoder," IEEE Journal of Solid-State Circuits, Vol. 30, No. 7, pp. 812-818, July 1995.

[15] S. Bitterlich and H. Meyr, "Efficient Scalable Architectures for Viterbi Decoders," in International Conference on Application Specific Array Processing (ASAP), Venice, Italy, October 1993.

[16] Stefan J. Bitterlich, Bert Pape, H. Meyr, “Area Efficient Viterbi-Decoder Macros," Proceedings of the European Solid-State Circuits Conference (ESSCIRC'94), Ulm, Germany, 1994.

[17] Lee-Fang Wei, "Trellis-Coded Modulation with Multidimensional Constellations," IEEE Transactions on Information Theory, Vol. IT-33, No. 4, July 1987.

[18] J. P. Kermoal, L. Schumacher, K. I. Pedersen, P. E. Mogensen, F. Frederiksen, "A Stochastic MIMO Radio Channel Model With Experimental Validation", IEEE Journal on Selected Areas in Communications, Vol. 20, No. 6, pp. 1211-1226, Aug. 2002.

[19] G. C Clark, Jr., and J. B. Cain, Error-correction coding for Digital Communications, plenum, 1981 